\title{
The Impact of the Patient's State on the Destination Care Unit Choice Within the Health Emergency Services Regional Network (HES-RN) in Morocco
}

\author{
Ibtissam Khalfaoui $^{1 *}$, Amar Hammouche ${ }^{2}$ \\ ${ }^{1}$ Research Team IMOSYS, Mohammadia School of Engineers, Mohamed V, Rabat, Morocco \\ ${ }^{2}$ PES, Research Team IMOSYS, Mohammadia School of Engineers, Mohamed V, Rabat, Morocco
}

Corresponding Author Email: khalfaoui.ibtissam.gip@gmail.com

https://doi.org/10.18280/ijsse.110419

Received: 4 January 2021

Accepted: 10 July 2021

\section{Keywords:}

care unit choice, decision support model, GIS, health emergency services regional network, patient's state of severity, qualified care units

\begin{abstract}
\end{abstract}
\section{INTRODUCTION}

In the pre-hospital emergencies, it is very important to transfer the patient as quickly as possible. To do this, it is first necessary to determine the patient's state of severity in order to list the qualified care units $(\mathrm{CUq})$ of destination able to receive and treat the patient correctly.

In this kind of urgent situations, time is of crucial importance. Indeed, if the patient's condition worsens, the patient's survival time interval may decrease and the choice of CUd may change accordingly.

In this study, we are interested in the choices of qualified CUs in the case of an Occurrence of events of Health Emergency (OHE), and according to the patient's level of severity. In general, medical staff -with patient's relatives' consent when possible - make decisions about the choice of CUd on behalf of their patients, unless the patient's condition is stable, patients make the final decision on the choice of hospital themselves.

The qualification of the CU of destination designated by $\mathrm{Q}_{\mathrm{CU}}$ is a very critical step in our study. However, this qualification depends particularly on the value of the patient's severity "Sp".

\section{LITERATURE REVIEW}

The patients' choices are impacted by infrastructural factors of health care quality, which includes availability and accessibility of providers, type and size of providers, experience, quality of the staff, cost of treatment along with available data, waiting time, and continuity and the quality of treatment [1].

As in ref. [2] 'Multispecialty hospital' and 'waiting time' are the most important factors for young age group to choose a hospital.

In the same research, 'Multispecialty hospital' is the most influencing factor for patients in the age group of 46-65 years and the 'Cost' is the most influencing factor in age group of above 65 .

Many variables influence consumers' choice of health care facilities. These variables can be characterized as care (quality, selection, cost), staff, physical facilities, clientele, experience, convenience (location), and institutional (reputation). The quality of medical care provided and the hospital staff are of particular importance. While the cost of care as a factor contributing to hospital choice is ranked relatively low in most studies in which it is included, it is mentioned more frequently than most factors, suggesting it is of greater importance than the face-value findings suggest, confirmed Gooding [3].

In their research, Adams et al. [4] added an important characteristic that would affect hospital choice, which is the patients' severity or complexity of illness. Since those more severely ill are more likely to need, or want, treatment with advanced technology and by highly skilled personnel, the researchers expected increased severity to be associated with an increased probability of choosing a larger, more sophisticated hospital.

In our study, we are interested in the choice of destination hospital, during a medical emergency, and we take into consideration the patient's state of severity while the OHE.

\section{METHODS}

It is obvious that the patient's state of severity has a very strong impact on the patient's transfer time. The more stable the patient's condition, the wider the range of care units.

We have already dealt with the different possible states of this severity as well as its criteria [5]. In this paper, and in addition to this study, we are interested in the dynamic evolution of these states and the decision to which CUd should the patient be transferred as quickly as possible. 


\subsection{Heuristic algorithm QQS-OHE (Quick Qualified Search-OHE)}

As mentioned in our previous study [5], and in order to find 1) the destination care units that are qualified to treat the OHE in question depending on the severity of the concerned patient and 2) the fastest possible path to transfer this one, between the OHE and the chosen CUd, we propose an improvement of our heuristic algorithm:

(1) Pinpoint the OHE location (geographical coordinates) and characterize it.

(2) Determine the type of severity g of this OHE and the qualification Qgj retained for this severity among $\mathrm{mg}$ qualifications of this type $\mathrm{g}$ (Figure 1).

(3) For the selected severity g, determine and classify, according to the qualification of this severity, the qualified CUds (as feasible points) to deal with the OHE in question (Figure 1).

(4) For each qualified CU, determine the path that will transfer the patient from the OHE location to it, as quickly as possible.

(5) Taking into account the severity of the considered OHE and the CU's qualification for this severity, determine the optimum torque (Qualified CU, Optimal path from $\mathrm{OHE}$ location to this $\mathrm{CU}$ ).

The implementation of step 1 is facilitated by the use of a GIS platform which has integrated functions for locating and visualizing places, in this case the OHE being processed. This is also the case for step 3 , which requires a ranking available to this platform. However, steps 2, 4 and 5 are somewhat complex.

The difficulties for steps 2 and 3 are respectively those of qualifying the OHE severity levels and determining the elements of qualification of a CUd.

As for step 4 and 5, we are faced with the problem of simultaneous search for the shortest paths (fast in our case) between different pairs of nodes; that of the OHE and that of a CUd selected from all of the qualified CU for that OHE.

In this work, we focus on these different steps.

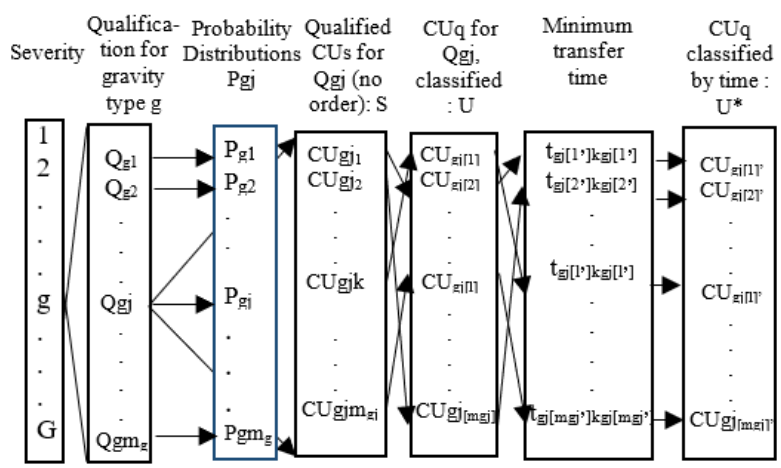

Figure 1. Classified CUq according to the OHE severity

We will deal with the issues in step 2 and 3 which are: 1) qualifying the severity of the patient's condition designated by $\mathrm{S}_{\mathrm{P}}$ and 2) qualifying the CU of destination designated by $\mathrm{Q}_{\mathrm{CUT}}$.

\subsubsection{Sp study}

It is obvious that if the patient's condition worsens, the patient's survival time interval may decrease and the choice of CUd may change accordingly. How then to deal with the issue of patient survival time following an OHE of a given severity?

By considering a patient as a human resource having a certain potential to produce something, we consider that this resource - during his production lifetime -1) remains available to produce as long as his state of health allows it (state of good functioning) and 2) may see this condition deteriorate to such an extent that it can temporarily no longer produce (fails 'down' and can be 'repaired') or may no longer be able to do so permanently.

To be able to answer the aforementioned question, we propose to characterize the uptime of a human resource using a reliability model. For a non-human production resource, one of the most widely used reliability models is that characterized by a negative exponential distribution. We are not going to venture to make the assumption that this would be the case for a patient because it would have to be validated; something that is beyond our reach in this study.

Nonetheless, and for the purpose of this paper, we assume that this would be the case, and we assume that this model only applies once the OHE occurs. In other words, we will use the reliability model as proposed (see Figure 2) as a "survival" model for the concerned patient:

Therefore, let:

Tgj the random variable representing the patient's survival time.

$\mathrm{f}$ (tgj) the probability density of Tgj.

$\tau_{\mathrm{gj}}$ a time offset from which Tgj is activated. $\tau_{\mathrm{gj}}$ is therefore the moment when the patient's vital prognosis is engaged.

$t_{\text {off }}$ is the OHE notification time.

$\lambda$ is the patient's condition deterioration rate.

$\mathrm{Q}_{\mathrm{g}}$ the random variable representing g- type severity qualification of the $\mathrm{OHE}$ having the probability distribution: $\left\{\mathrm{Q}_{\mathrm{g} 1}, \mathrm{Q}_{\mathrm{g} 2}, \ldots, \mathrm{Q}_{\mathrm{gj}}, \ldots, \mathrm{Q}_{\mathrm{gmg}}\right\} \rightarrow\left\{\mathrm{P}_{\mathrm{g} 1}, \mathrm{P}_{\mathrm{g} 2}, \ldots, \mathrm{P}_{\mathrm{gj}}, \ldots, \mathrm{P}_{\mathrm{gmg}}\right\}$ with $P_{g j}$ being the survival probability associated with $Q_{g j}$.

Then we can, from this survival model, estimate, among other things, the survival probabilities of the patient affected by the considered OHE and the time intervals of patient survival following an OHE as follows:

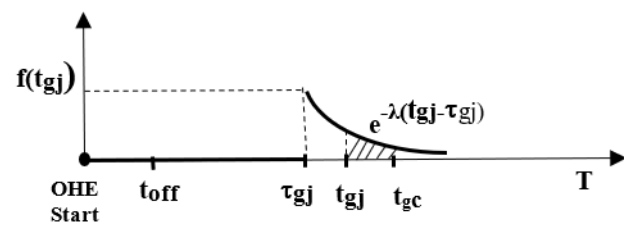

Figure 2. Patient's state of health deterioration density of probability

Survival probabilities. At the initial time of OHE, the patient's state of health $\mathbf{S}(0)$ undergoes a first degradation due to this OHE. To simplify, we suppose that this state remains stable until the moment $T_{g j}=\tau_{g j}$ when it starts to degrade a second time in an exponential way according to the probability density $\left(=\lambda \mathrm{e}^{-\lambda(\mathrm{tgj}-\mathrm{\tau g})}\right)$ of Figure 2 . Let us denote this state at time t by $\mathbf{S}(\mathrm{t})$ and $\mathrm{OHEQ}_{\mathrm{gj}}$ the event 'OHE with $\mathrm{Q}_{\mathrm{gj}}$ severity'. Then the patient's survival probability at the OHE is:

$$
\begin{aligned}
& P_{g j}\left(t_{g j}\right)=\operatorname{Pr}\left(S\left(t_{g j}\right) \cap O H E Q_{g j}\right) \\
& =\operatorname{Pr}\left(O H E Q_{g j}\right) \times \operatorname{Pr}\left(\boldsymbol{S}\left(t_{g j}\right) \mid O H E Q_{g j}\right)=P_{g j} \times \\
& \operatorname{Pr}\left(T_{g j}>t_{g j}\right) \\
& =P_{g j} \times\left(1-\operatorname{Pr}\left(T_{g j}<t_{g j}\right)\right) \\
& =\left[\begin{array}{ll}
P_{g j} & \text { if } t_{g j} \leq \tau_{g j} \\
P_{g j} \times e^{-\lambda\left(t_{g j}-\tau_{g j}\right)} & \text { if } t_{g j}>\tau_{g j}
\end{array}\right.
\end{aligned}
$$


Time intervals. The overriding objective for an emergency team treating a given $\mathrm{OHE}$ is to be able to transfer the affected patient within the minimum possible time that would maximize the likelihood of survival. According to our model, this duration is $d_{g j . m i n}=t_{g j . m i n}-t_{o f f} \leq \tau_{g j}-t_{o f f}$. However, and for various hazards, this transfer time could reach higher values which risk reducing the probability of patient survival to "hopeless" values.

We suppose that the maximum duration for which this probability becomes 'desperate' is $d_{g j . m a x}$ such that $P_{g j}\left(t_{g j . m a x}\right)=$ $\mathrm{P}_{\mathrm{gj.D}}$ with $\mathrm{t}_{\mathrm{gj} \text {.max }}=\mathrm{d}_{\mathrm{gj.max}}+\mathrm{t}_{\text {off. }}$. Then, we can retain the tolerance interval $\mathrm{TIt}=\left[\mathrm{d}_{\mathrm{gj} \cdot \mathrm{min}}, \mathrm{d}_{\mathrm{g} \text {.max }}\right]$ as the criterion interval for choosing the CUd of the concerned patient. On the other hand, and once $\mathrm{P}_{\text {g..D }}$ is set for an $\mathrm{OHE}$ with a given severity qualification $\mathrm{Q}_{\mathrm{g} j}$, we can determine $\mathrm{t}_{\mathrm{gj} \text {.max }}$ by inverting the patient survival degradation distribution function as defined above:

$$
\begin{array}{lrl} 
& & F\left(t_{g j_{\text {max }}}-\tau_{g j}\right)=P_{g j_{D}} \\
\text { So } & t_{g j_{\max }}-\tau_{g j}=F^{-1}\left(P_{g j_{D}}\right) \\
\text { and } & t_{g j_{\max }}=F^{-1}\left(P_{g j_{D}}\right)+\tau_{g j}
\end{array}
$$

Having retained a negative exponential model for the patient severity degradation, we have:

$$
\begin{gathered}
F\left(t_{g j_{\max }}-\tau_{g j}\right)=1-e^{-\lambda\left(t_{g j_{\max }}-\tau_{g j}\right)}=P_{g j_{D}} \\
\Rightarrow F\left(t_{g j_{\max }}-\tau_{g j}\right)=1-e^{-\lambda\left(t_{g j_{\max }}-\tau_{g j}\right)}=P_{g j_{D}} \\
\text { And } d_{g j_{\max }}=\tau_{g j^{j}}(1 / \lambda) \ln \left(1-P_{g j_{D}}\right)-t_{o f f}
\end{gathered}
$$

\subsubsection{Q $\mathrm{CU}$ study}

In general, several factors influence patients' decisions about the choice of care units. The selection criteria may vary depending on the patient's needs [6]. Identifies a selection criteria for each type of service. The first five criteria used for each type of service are summarized as follows:

- General care services: 1) proximity to residence / convenience, 2) physician recommendation, 3) past hospital experience, 4) familiarity with hospital staff, 5) quality of facilities physical (building structure, cleanliness, access to elevators and environment).

- Specialized care services: 1) availability of qualified specialist doctors, 2) doctor's recommendation, 3) past hospital experience, 4) availability of the best equipment and technologies, 5) proximity to the residence.

- Emergency care services: 1) proximity to the residence,

2) past hospital experience, 3 ) doctor's recommendation,

4) be familiar and satisfied with hospital staff, 5) availability and quality of facilities physical.

According to Akinci et al. [6], hospitals should document health outcomes and they should be willing to make outcome data public to enable patients to make informed decisions about their hospital choice.

In our study, we are only interested in the choices of qualified CUs in the case of an OHE and according to the patient's level of severity.

The qualification of the CUd designated by $\mathrm{Q}_{\mathrm{CU}}$ is a very critical step in our study. However, this qualification depends particularly on the value of Sp.
The choice of qualified CUds for an OHEQ $\mathrm{gj}$ is broadened or limited, depending on Sp value.

Once the OHE takes place, the rescue team arrives at the scene and a medical diagnosis is immediately made. This is done based on the patient's symptoms as well as the results of clinical examinations performed (see Figures 3 and 4). The accuracy of the medical diagnosis depends on the availability and quality of the ambulance equipment, as well as the skill of the rescuers.

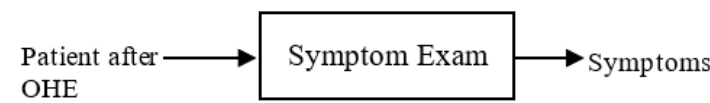

Figure 3. SADT diagram of symptom exam

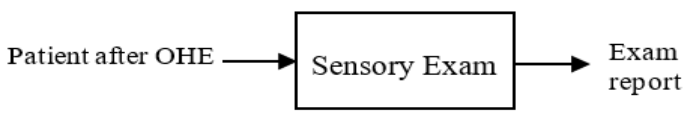

Figure 4. SADT diagram of sensory exam

In such a circumstance, the access to the patient's medical records is of utmost importance. Several information concerning his antecedents (pathologies, allergies, treatments followed, blood group, etc.), his attending physicians, his past diagnoses, in particular those carried out under similar conditions, can be of great use in the development of a diagnosis and a rigorous action plan (see Figure 5).

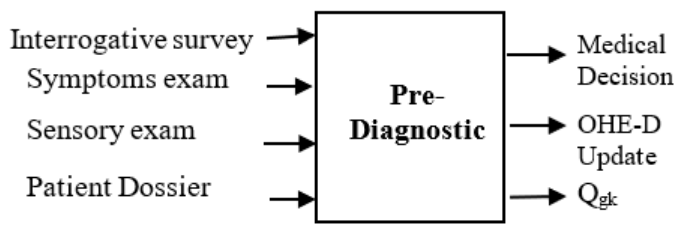

Figure 5. SADT A-0 diagram of the patient's diagnosis

If the patient's condition allows it, he gives his consent to the rescuer to access his health data. Otherwise, if the patient is unconscious, the medical staff contacts the SAMU (the HES-RN's Control centre), informs them of the national ID card code and the patient's level of severity and requests access to the file.

This is immediately accessible if the level of severity is greater than or equal to the $\mathrm{g}_{\text {critical }}$ threshold (severity from which the patient's life is in danger).

Once the diagnosis is made, the level of severity is determined and any decision making becomes easier and more obvious.

All the information collected on the patient as well as the decisions taken by the rescuers will be communicated to the 48SAMU and recorded on the "OHE-D" and on the "Unique Patient Dossier" [5] so that the CUd or any other care unit can access it, to eventually use them.

If the patient's condition requires it, the medical team performs an initial intervention on site, in order to stabilize or even improve the patient's probability of survival. The patient is then transferred to the CUq of destination. Throughout his transfer, he continues to receive the necessary care. If the patient's severity level decreases from $g$ to $g-1$ or less, then the CUd's TIt interval of choice will expand to other CUd and the CUq originally chosen as the destination may change.

If, on the other hand, the level of severity increases then the 
interval TIt is reduced and the probability of survival may become "hopeless" if the transfer time exceeds $d_{\text {gj.max }}$ (see Figure 6).

As the patient's severity level is dynamic and changes over time, it should be remembered that the course of action or any decision is not static and is subject to change at any time.

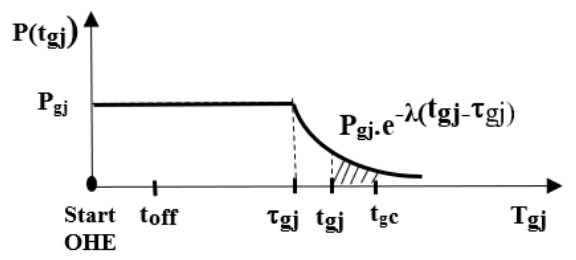

Figure 6. Patient's probability of survival

We conclude this $\mathrm{Q}_{\mathrm{Cu}}$ study by saying that the patient's state severity can be essentially identified using1) the established diagnosis: $\mathrm{Q}_{\mathrm{gk}}=\mathrm{f}$ (Medical exams: symptoms, sensory exam, Patient Dossier, ...), and 2) the severity repertoire and 3) modelling the patient survival distribution for a given severity Qgk.

Once the qualification of the patient's severity is determined, the medical staff should determine, based on the CUd repertoire of standard hospital processes, the necessary hospital processes to treat the concerned patient (See Figure 7).

This poses the problem of CU's qualification that should receive the concerned patient. To solve such a problem, we should answer a series of questions regarding the management of the OHE in question:

(1) Why? The life of the patient affected by the OHE in question could be in danger if this OHE is not managed or improperly managed.

(2) What? Diagnosis of the state of health of the concerned patient; Transfer of this patient from the OHE location as quickly as possible, to a CUd as qualified as possible:

- The severity $\mathrm{Q}_{\mathrm{gk}}$ of the $\mathrm{OHE}$ in question should be determined from the severity repertoire while relying on the medical diagnosis made.

- The fastest patient's transfer requires the search for the fastest dynamic routing of this patient along a route determined by the heuristic algorithm QQS-OHE proposed above.

- The CUs' classification according to their qualifications requires a normalization and standardization of the management systems of these CUs as well as their hospital processes.

(3) How? The hospital processes $P_{g j}$ necessary to manage the OHE in question depending on its severity ' $\mathrm{Q}_{\mathrm{gj}}$ '. The CUd must have; among others, these processes in their processes repertoire.

(4) With what? The human, hardware, and software resources required to treat the $\mathrm{Q}_{\mathrm{gj}}$ patient's severity, using the $\mathrm{P}_{\mathrm{gj}}$ process.

(5) When? The criticality of the time needed to manage an $\mathrm{OHE}$ is, mainly, a function of this OHE severity ' $\mathrm{Q}_{\mathrm{gk}}$ ', and the CU's qualification selected to process it. Depending on this OHE severity, a minute could make the difference between the patient's life and death.

The answers given to these questions mainly form the basis for the mapping of Figure 7, which we propose and which are able to lead to standardized qualifications of the CUd. Only such qualifications could guarantee the deployment and perrenization of the quality assurance of the OHE management within the HES-RN.

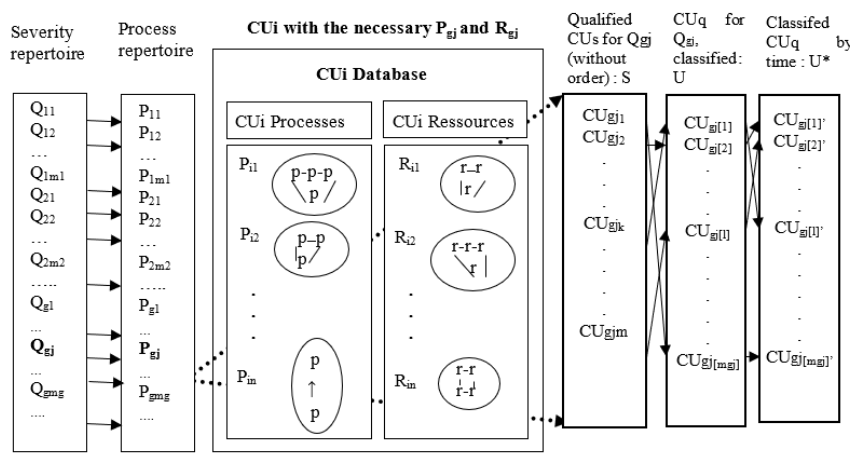

Figure 7. Mapping to find qualified CUds based on severity and process repertoires and the CUi database

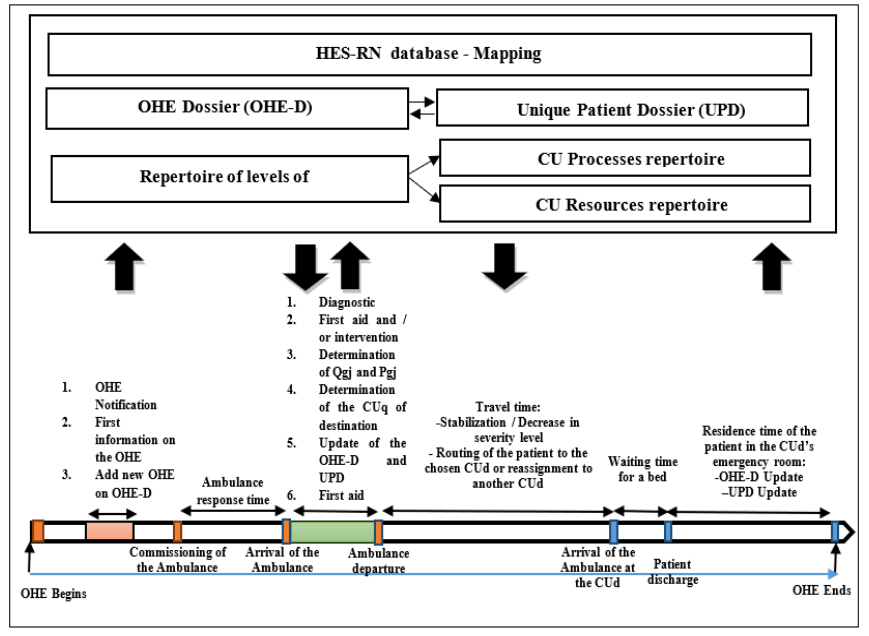

Figure 8. OHE life cycle

From Figure 7, the list of qualified CUs is identified based on the HES-RN USi database, with their processes and resources (represented in the form of graphs), and according to the OHE severity Qgj, referenced in the OHEs severity repertoire.

These repertoires represent essential references for the hospital network in determining CUq. The combination of both repertoires and the databases of the network's map, constitutes a rich and reliable support for the HES-RN in the management of urgent medical events.

Thus, the OHE life cycle becomes as shown in Figure 8.

In order to synthesize this part, we present in Figure 9 a simplified global model of the HES-RN, showing the graph model of it as well as the main part of its infrastructure.

This figure also includes the routing for a given OHE.

With:

MERS: Mobile Emergency and Resuscitation Service

According to Figure 9, several searches are being developed using the HES-RN infrastructure, in particular that of $\mathrm{Q}_{\mathrm{CU}}$.

Based on this HES-RN model and its infrastructure and in order to achieve a consistent and efficient $\mathrm{Q}_{\mathrm{CU}}$ result, it is necessary to first define the research criteria. By implementing this qualification research using the GIS platform, it is also important to note that during our request for this research using the HES-RN GIS map, carried out on this platform, several criteria are likely to change over time, such as availability of beds, availability of attending physicians, availability of equipment, etc. 
Thus, an update of the set S of qualified CUs is essential.

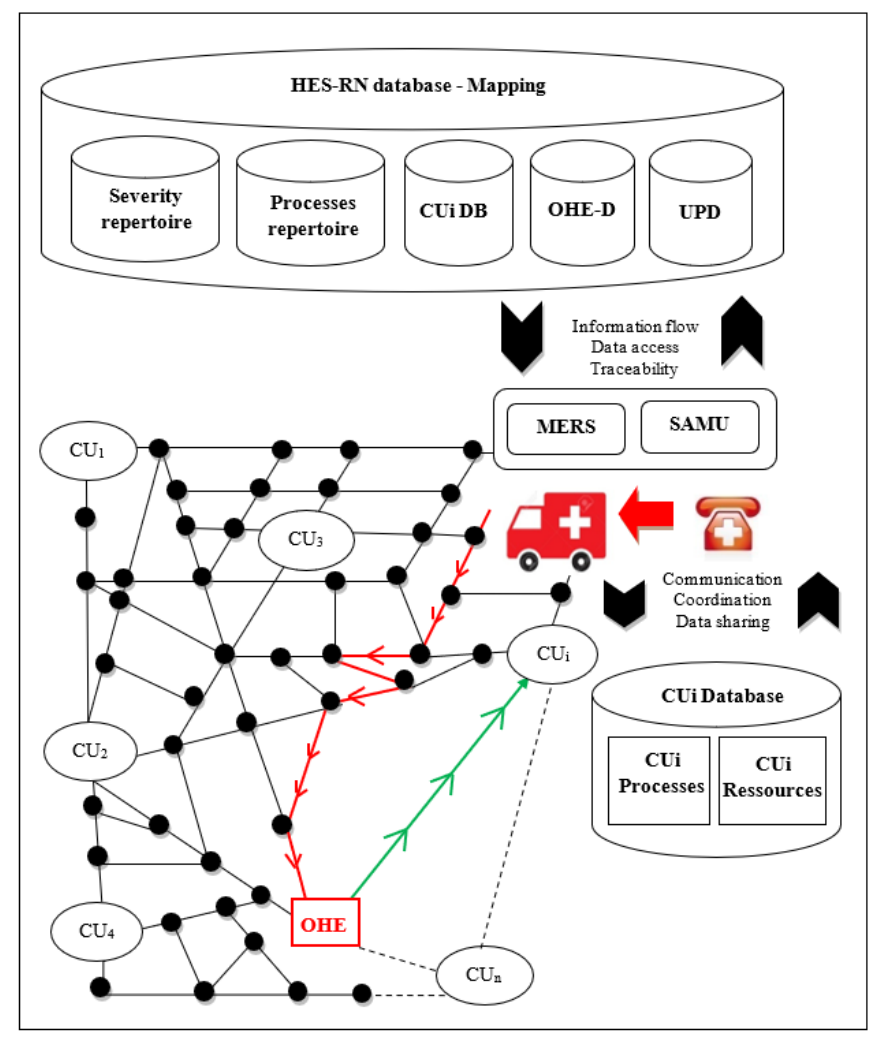

Figure 9. HES-RN model and infrastructure

The GIS implementation of this search can be done using:

A new tool for "Searching for qualified CUds". From the HES-RN's database that we built using this platform, we were able to create a search interface, thus facilitating the execution of the query task.

The VBA code produced in ArcMap, allows us to create and display, on the map toolbar, a new search tool "Qualified Cud", allowing the user to enter the query data and to interact with the HES-RN CU database (see Figure 10).

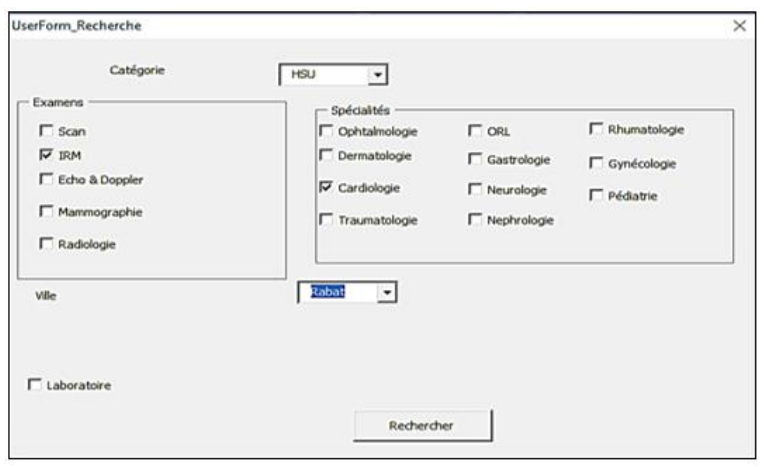

Figure 10. Qualified CUds search GIS interface

The ArcGIS ModelBuilder module. This tool, which is part of ArcMap, also allows us to graphically model the search query for qualified CUds that can respond to an $O H E Q_{g j}$. It makes it possible to build a model of a given search by assembling and "piping" different tools integrated into ArcMap or developed, if necessary, from such tools or from ArcMap bricks.

For example, the "Select (Analysis)" tool is a simple means that allows to perform various queries against the CUs database in the studied region. As shown in Figures 11 and 12, the search criteria are defined in the "Expression" box of the Select tool using "Query Builder".

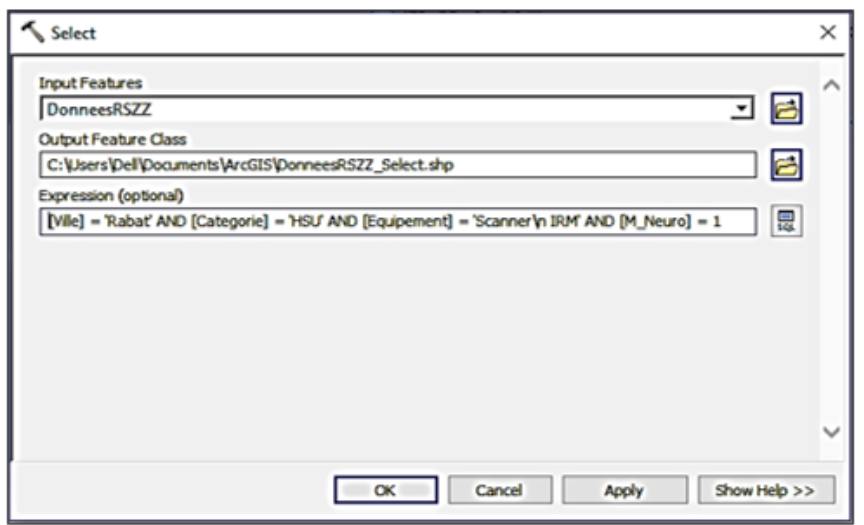

Figure 11. ModelBuilder's "Select" tool

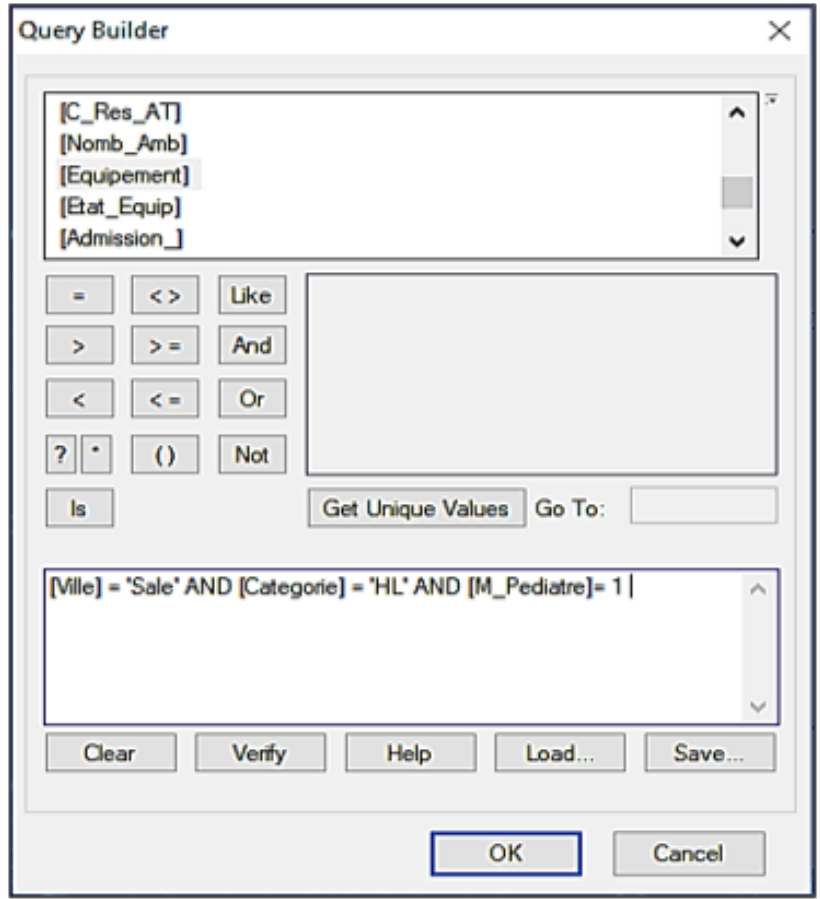

Figure 12. Criteria in the "Query Builder" window

The progress of such a search takes place once the patient's state of severity " $g$ " as well as the qualification of this state "Qgj" have been determined. Thus and for a given Qgj we have to:

- Query the HES-RN database to determine all the CU with the necessary and sufficient Emergency Hospital Processes (EHP) (qualified CU) to treat the OHE according to the qualification of its severity "Qgj".

Let $\mathrm{S}$ be the nonempty set of these qualified CU for "Qgj", with $\mathrm{S}=\{$ CUqi $/ \mathrm{i}=1, \mathrm{n}\}$.

Having solved the problem of qualifying the destination CU for a qualification of a given patient's state severity, it will be easy for us to continue the development of this QQS-OHE and the steps 3 and 4 become:

- Classify S according to the CUi qualification for the "Qgj" severity state.

Let $\left.\left.\mathrm{S}^{*}=\left\{\mathrm{CUq}_{<1>}>\mathrm{CUq}_{<2>}>\mathrm{CUq}_{<3>}\right\rangle \ldots\right\rangle \mathrm{CUq}_{<\mathrm{n}>}\right\}$

Such that $<j>$ is the qualification index of the CUq which 
varies from 1 (the most qualified $\mathrm{CU}$ ) to $\mathrm{n}$ (the least qualified $\mathrm{CU}$ ) (' $)$ ' being the descending order of the CUq qualification in $\mathrm{S} *$ ).

- Find all the routes that can connect the OHE location to these qualified CUs.

Let $\mathrm{Ri}=\left\{\mathrm{r}_{\mathrm{ik}} / \mathrm{k}=1 \ldots \mathrm{m}\right\}$ be the non-empty set of connection routes to $\mathrm{CUi}$ and

$t_{i k}=$ duration of road $r_{i k}=$ (total length of fluid type travels) / vmax + (total length of slow type travels) / v1 + (total length of average type travels) / v2 + (total length of stop \& go type travels) / vmin.

- Let $\mathrm{t}_{\mathrm{iki}}=\min \left\{\mathrm{t}_{\mathrm{ik}}, \mathrm{k}=1 \ldots \mathrm{m}\right\}$ for $\mathrm{k}=\mathrm{k}_{\mathrm{i}}$.

So $r_{i k i} \in \mathrm{Ri}$ with $t_{i k i}$ journey time is the fastest route associated with CUi.

- Classify the CUi of $\mathrm{S}$, in ascending order of the $\mathrm{t}_{\mathrm{iki}}$ in the new set $\mathrm{U}^{*}=\left\{\mathrm{CUq}_{<1>}>\left\langle\mathrm{CUq}_{<2>},\left\langle\mathrm{CUq}_{<3}\right\rangle,\langle\ldots\right.\right.$ $\left.\left\langle\mathrm{CUq}_{<\mathrm{n}>}\right\rangle\right\}$ with $\langle$ being the increasing order of proximity in time, of the CUq to the OHE location, and $t_{i k i}$ : the minimum transfer time from the OHE location to the CUdi along the route $\mathrm{k}_{\mathrm{i}}$.

This classification allows us to establish the following mapping between $\left.\left.\left\{\mathbf{t}_{[\mathbf{i}]}\right]^{\prime} \mathbf{k}\right]\right]^{\prime} \mathbf{i}=\mathbf{1}, \mathbf{n}$ with ranking \langle\} and $U *$ (Figure 13).

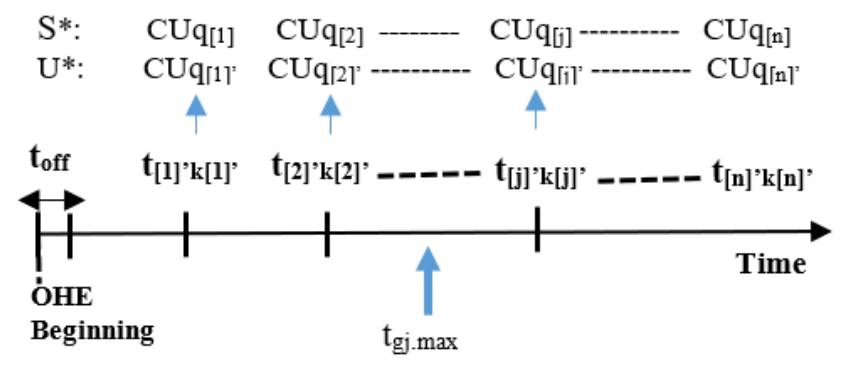

Figure 13. Minimum transfer times and corresponding CU

\section{DISCUSSION}

In this paper, we presented a proof of concept facilitating decision-making in medical emergency situations, whether in determining the patient's level of severity or in the choice of the destination care unit. Hence, we have focused on developing the steps of our heuristic algorithm QQS-OHE to carry it out.

This allowed us to conclude on the choice of the different candidate CUq to receive the patient, which takes into account both the CU qualification and the OHE severity:

- Candidate CUqs are those whose minimum transfer times $\mathrm{t}[\mathrm{j}]]^{\prime} \mathrm{k}[\mathrm{j}]$ 'are $\leq \mathrm{t}_{\mathrm{gj} \text {.max }}$. In other words, $\mathrm{t}_{\mathrm{gj} \text { max }}$ constitutes a cursor for the choice of these CUq.

- The priority choice of destination CUqs for the patient is $\mathrm{CUq}_{[\mathrm{j}]}$, which corresponds to $\left.\left.\mathrm{t}_{[\mathrm{j}]}\right]^{\prime} \mathrm{kj}\right]^{\prime} \leq \mathrm{t}_{\text {gimax }}$ while taking into consideration its classification in the set $\mathrm{S} *$.

Then, we can distinguish three possible scenarios:

- In the optimistic case, the destination CU is the one ranked first in the both sets $\mathrm{S} *$ and $\mathrm{U} *$.

- In the pessimistic case, the destination $\mathrm{CU}$ is the one ranked first in the set $\mathrm{U}^{*}$ and last in the set $\mathrm{S} *$.

- In the general case, the destination CU is the one whose minimum transfer time is less than or equal to $t_{\text {gimax. }}$.
Taking into account the results of these two studies, the algorithm becomes as shown in Figure 14 below:

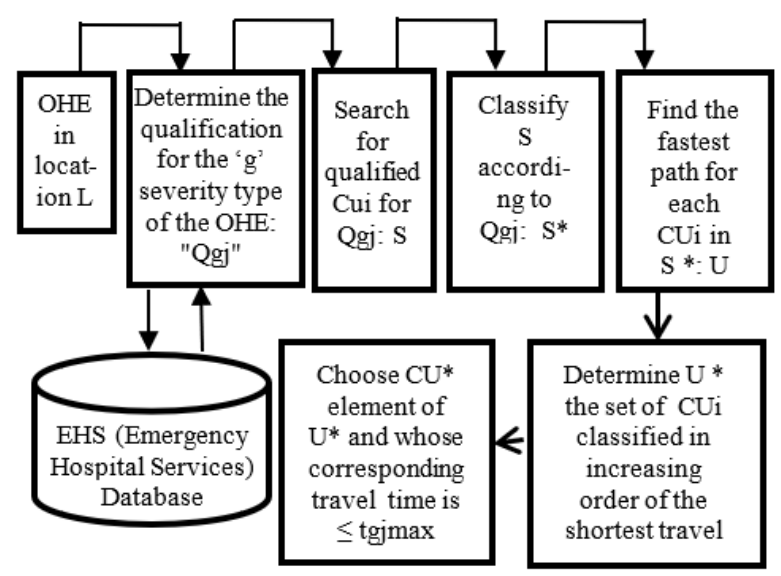

Figure 14. The heuristic algorithm QQS-OHE steps

Concerning the implementation and the simulation of this heuristic algorithm and due to data limitations, we have, for now, done them only partially using a GIS platform. Their completion is being worked on

\section{CONCLUSIONS AND PERSPECTIVES}

The decision within a system as complex as that of HES$\mathrm{RN}$ is combinatorial and vague in nature, whether at the medical level or at the logistical level.

We have proposed a heuristic algorithm to manage the OHE-Process aiming for the best possible choice of the patient's qualified destination care unit, and the transfer of this patient to this CUd as quickly as possible depending on the severity type of this one.

In this paper, and for a given OHE, we focused on critical decisions concerning: 1) the pre-hospital management of the patient's condition at the OHE site and / or during his transfer to a destination $\mathrm{CU}, 2$ ) the qualification of that $\mathrm{CUd}$, and 3) the choice of it with such a qualification, while maintaining the patient's probability of survival as high as possible.

The choice of the latter with such a qualification, is made by relying on the "Resources" and "Process" repertoires of the studied HES-RN CUs.

As to our future works, we are working on implementing this decision support model in the studied HES-RN.

\section{ACKNOWLEDGMENT}

This work is part of the research project $\mathrm{N}^{\circ}$ SIT05 / 09, funded by Mohammed V University, within the framework of the agreement between this University and Ibn Sina Hospital Center.

\section{REFERENCES}

[1] Chauhan, V., Sharma, A., Sagar, M. (2021). Exploring patient choice in India: A study on hospital selection. International Journal of Healthcare Management, 14(2): 610-620.

https://doi.org/10.1080/20479700.2019.1679520 
[2] Bhangale, V. (2011). Marketing of healthcare services in India: A study on factors influencing patients' decision making on choice of a hospital. Journal of Management \& Marketing in Healthcare, 4(4): 229-233. https://doi.org/10.1179/175330311X12943314049376

[3] Gooding, S.K.S. (2000). Quality, sacrifice, and value in hospital choice: A comparative study of rural and suburban consumer perspectives. Journal of Hospital Marketing, 13(2):

23-42. https://doi.org/10.1300/J043v13n02_03

[4] Adams, E.K., Houchens, R., Wright, G.E., Robbins, J. (1991). Predicting hospital choice for rural Medicare beneficiaries: the role of severity of illness. Health Services Research, 26(5): 583-612.

[5] Khalfaoui, I., Hammouche, A. (2020). Modelling and optimizing health emergency services: A regional study case. International Journal of Healthcare Management, 112. https://doi.org/10.1080/20479700.2020.1801163

[6] Akinci, F., Esatoglu, A.E., Tengilimoglu, D., Parsons, A. (2004). Hospital choice factors: a case study in Turkey. Health Marketing Quarterly, 22(1): 3-19. https://doi.org/10.1300/j026v22n01_02

\section{NOMENCLATURE}

$\mathrm{f}$ (tgj) The probability density of Tgj

$\tau_{\mathrm{gj}} \quad$ A time offset from which Tgj is activated

$t_{\text {off }} \quad$ The OHE notification time

$\lambda \quad$ The patient's condition deterioration rate

g The severity type of the OHE

$\mathrm{Q}_{\mathrm{g}} \quad$ The random variable representing g- type severity qualification of the $\mathrm{OHE}$

$\mathrm{d}_{\text {gj.min }}$ The minimum possible time that would maximize the likelihood of patient's survival

$\mathrm{P}_{\mathrm{gj}} \quad$ Patient's survival probability associated with $\mathrm{Q}_{\mathrm{gj}}$

S The nonempty set of qualified CU for "Qgj"

$\mathrm{g}_{\text {critical }}$ severity from which the patient's life is in danger

S* Elements of S classified according to Qgj

$\mathrm{U} \quad$ Set of fastest paths for each CUi in $\mathrm{S} *$

$\mathrm{U}^{*} \quad$ Set of CUi classified in increasing order of the shortest travel times

$t_{i k} \quad$ Duration of road $r_{i k}$

tgc Time from which the patient's probability of survival tends to 0

TIt Tolerance interval

$\mathrm{d}_{\mathrm{gj} \text {.max }}$ The maximum duration for which the probability of survival becomes 'desperate' 